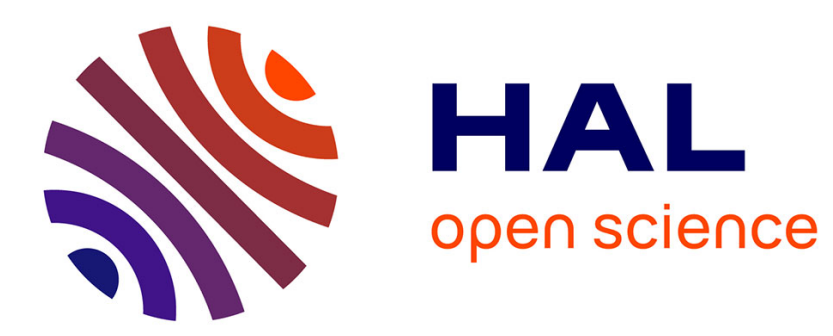

\title{
Efficient field emission from structured gold nanowire cathodes
}

\author{
A. Navitski, G. Müller, V. Sakharuk, T.W. Cornelius, C. Trautmann, S. \\ Karim
}

\section{- To cite this version:}

A. Navitski, G. Müller, V. Sakharuk, T.W. Cornelius, C. Trautmann, et al.. Efficient field emission from structured gold nanowire cathodes. European Physical Journal: Applied Physics, 2009, 48 (3), pp.1-7. 10.1051/epjap/2009167 . hal-00530827

\section{HAL Id: hal-00530827 https://hal.science/hal-00530827}

Submitted on 30 Oct 2010

HAL is a multi-disciplinary open access archive for the deposit and dissemination of scientific research documents, whether they are published or not. The documents may come from teaching and research institutions in France or abroad, or from public or private research centers.
L'archive ouverte pluridisciplinaire HAL, est destinée au dépôt et à la diffusion de documents scientifiques de niveau recherche, publiés ou non, émanant des établissements d'enseignement et de recherche français ou étrangers, des laboratoires publics ou privés. 


\title{
Efficient field emission from structured gold nanowire cathodes
}

\author{
A. Navitski*, G. Müller, V. Sakharuk \\ FB C Physik, Bergische Universität Wuppertal, D-42119 Wuppertal, Germany \\ T. W. Cornelius, C. Trautmann \\ GSI Helmholtzzentrum für Schwerionenforschung, D-64291 Darmstadt, Germany \\ S. Karim \\ PINSTECH, Islamabad, Pakistan
}

\begin{abstract}
Regular patch arrays of random gold nanostructures were fabricated by electrochemical deposition of nanowires in ion track-etched templates. During ion irradiation with $\mathrm{GeV}$ ions of fluence $10^{6}, 10^{7}$, or $10^{8} \mathrm{~cm}^{-2}$, a shadow mask was used resulting in templates structured with square arrays of $50 \mu \mathrm{m}$ holes and 100 or $150 \mu \mathrm{m}$ pitch. The Au nanowires grown in the track-etched pores had a length of 7-28 $\mu \mathrm{m}$ and a diameter of $\sim 300 \mathrm{~nm}$, and were either solitary or clustered after template dissolution. The structured wire ensembles were systematically investigated with scanning electron and field emission scanning microscopy. Field emission with about $90 \%$ efficiency was achieved for wide-spaced patch arrays with medium and high number of Au nanowires at $1500 \mathrm{~V}$ for $20 \mu \mathrm{m}$ anode distance. The current carrying capability of the patches strongly varied between $40 \mathrm{nA}$ and $90 \mu \mathrm{A}$. The corresponding processing effects are correlated to adsorbates and nanostructural changes of the wires which give suitable hints for the optimization of structured Au nanowire cathodes.
\end{abstract}

Keywords: gold nanowires; structured cathode; ion-track template technique; scanning microscopy; field emission.

PACS: 79.70.+q

*Corresponding author: Aliaksandr Navitski

contacte-mail:navitski@physik.uni-wuppertal.de

tel. / fax: + 49202 439-3492 /-28 11 


\section{Introduction}

In the last ten years cold field emission (FE) cathodes based on carbon nanotubes (CNT) have been widely studied due to their simple fabrication and large electric field enhancement $[1,2]$. The applicability of CNT cathodes in vacuum nanoelectronic devices, however, is still limited by the homogeneity, stability and controllability of the current in defined FE arrays [3-5]. Metal nanowires (NW) with high aspect ratio (AR) and high conductivity fabricated by the ion-track template technique $[6,7]$ provide an interesting alternative. The method allows in particular the control of the number density of NW by the fluence applied during template irradiation, whereas the wire diameter and length are controlled by time of the track etching and electrochemical deposition, respectively [8]. Based on the promising FE results obtained for randomly distributed copper [9], gold-coated nickel [10], and gold NW [11] in diode configuration, this study focuses on the development of structured cathodes with defined Au-NW patch arrays which might be suitable for triode applications with low gate currents.

Here we report on first square arrays of Au-NW patches with two pitch sizes which were obtained by using a structured metallic shadow mask during the heavy ion irradiation of the polycarbonate template foil. The influence of the number density and aspect ratio of the NW on the morphology of the free-standing solitary or clustered nanostructures of the resulting patch arrays was systematically investigated by means of scanning electron microscopy (SEM) and correlated to their FE performance as measured with a field emission scanning microscope (FESM) [12]. Moreover, the effective field enhancement factor was derived from locally measured current voltage (I-V) curves. For selected AuNW patches the maximum achievable current and resulting changes of the corresponding nanostructures were also investigated. The impact of these results on the possible optimization of AuNW cathodes for FE triodes will be discussed.

\section{Cathode fabrication and measurement techniques}

The successive fabrication steps of structured Au-NW cathodes are shown in figure 1. At first templates were produced by exposing $30 \mu \mathrm{m}$ thick polycarbonate foils (Makrofol N, Bayer AG) through a $200 \mu \mathrm{m}$ thick metallic mask (brass MS63) to energetic heavy ions. The irradiation 
experiment was performed at the UNILAC accelerator facility (GSI, Darmstadt) using $2.6 \mathrm{GeV}$ U ions and a fluence of $10^{6}, 10^{7}$, or $10^{8} \mathrm{~cm}^{-2}$. The metallic mask contains four square arrays of round holes of $\varnothing 50 \mu \mathrm{m}$ and a pitch $P$ of 100 or $150 \mu \mathrm{m}$ (two arrays each) (see figure 2). The ions create damage zones in the foils which were selectively etched as described earlier [6]. The resulting polycarbonate templates provide regular patch arrays (10000 or $4444 / \mathrm{cm}^{2}$, respectively). The patches contain randomly distributed but parallel-oriented cylindrical pores with a diameter D of about $300 \mathrm{~nm}$. For the wire growth, one side of the templates were sputter-coated by an Au layer $(\sim 20 \mathrm{~nm})$, which was further enhanced by an additional electroplated Cu layer (5-10 $\mu \mathrm{m}$, Cupatierbad, Riedel GmbH). This conductive backing layer with improved mechanical stability served as a substrate of the cathode. The deposition of $\mathrm{Au}$ into the pores was performed in a galvanic cell using a potassium dicyanoaurate (I) solution (Puramet 402, Doduco $\mathrm{GmbH}$ ) with $10 \mathrm{~g} /$ of gold at $50{ }^{\circ} \mathrm{C}$ and a gold rod as anode [8]. The length of the wires and, thus, their aspect ratio was controlled by deposition time and by recording the deposited charge. After the dissolution of the template in a $\mathrm{CH}_{2} \mathrm{Cl}_{2}$ solution, regular patch arrays of free-standing nanostructures on $\mathrm{Cu}$ substrates were achieved as shown in figure 2. Finally, all samples were carefully assembled on aluminium cathode holders of about $8 \mathrm{~mm}$ in diameter. The main parameters of the fabricated structured Au-NW cathodes are summarized in table 1.

The morphology of typical Au-NW structures before and after the FE measurements was investigated by SEM (Phillips XL30 at GSI and Phillips XL30S at Univ. Wuppertal). A FE sites distribution from the whole Au-NW cathodes (xy-surface tilt-corrected with respect to a flattened conical anode of $160 \mu \mathrm{m}$ diameter to achieve a constant gap $\Delta z$ within $\pm 1 \mu \mathrm{m}$ for the full scan area of $50 \mathrm{~mm}^{2}$ ) was non-destructively imaged with the FESM in vacuo $10^{-7} \mathrm{~Pa}$ by means of a regulated voltage supply (FUG HCN250M5000 with PID) controlled by the FE current (Keithley 610C) as described elsewhere [12]. After in-situ replacement of the conical by a needle anode (both made from tungsten-tantalum alloy 90:10) with a tip apex radius $R_{a}$ of $15 \mu \mathrm{m}$, the alignment, homogeneity and efficiency of FE from selected patch arrays were checked and the integral current of arbitrarily chosen Au-NW patches was measured. Sharper W tip anodes $\left(R_{a}<3 \mu \mathrm{m}\right)$ were used to resolve FE from single nanostructures. In comparison to unstructured cathodes it is remarkable that the array structure enabled us to correlate the FESM and SEM results for better understanding of properties of the nanostructures. 
The macroscopic electric field $E$ was calculated as ratio of applied voltage to an effective distance $d$ between a tip anode and a relevant emitter. This distance $d$ was determined for each FE site by the linear extrapolation of PID-regulated $V(z)$ curve (for $1 \mathrm{nA}$ fixed FE current) to zero voltage, which corresponds to contact between anode and emitter. The effective field enhancement factor $\beta_{\text {eff }}$ of the patches were derived from the measured I-V data using modified Fowler-Nordheim (FN) equation and assuming a work function of $4.9 \mathrm{eV}$ for $\mathrm{Au}$. It should be mentioned that for $d$ values close to or smaller than the mean NW length, the effective field enhancement factor $\beta_{\text {eff }}$ is not only given by the intrinsic field enhancement of the NW, but is also influenced by the $d$ value as suggested by the two region FE model of Zhong et al. [13]. The influence of the electrode gap geometry including the real shape of anode or gate on the field emission parameters should be taken into account for triode device development.

\section{Results and discussion}

Quite different free-standing nanostructures were obtained in the patch arrays of the Au-NW cathodes as shown in figure 3. Depending on the number density and aspect ratio, the wires are either solitary vertically-aligned or they agglomerate into conical clusters. Solitary wires are typical for low number density $\left(10^{6} \mathrm{~cm}^{-2}\right.$, cathode A) and for short $(<10 \mu \mathrm{m}) \mathrm{NW}$ at medium $\left(10^{7} \mathrm{~cm}^{-2}\right.$, cathode B) and high number density $\left(10^{8} \mathrm{~cm}^{-2}\right.$, cathode D). In contrast, most of the long NW at medium and high number density are agglomerated into conical clusters probably by bending during the wet dissolution process of the polymer template. Clustering is dominant for NW of high AR (high ductility) and when the average wire distance is much smaller than their length. It is remarkable that there are only a few clusters per patch for medium but many competing clusters for high number density of NW. It is of course difficult to predict the form of clusters especially in case of few clusters per patch, but some control is still possible by adapting NW density and AR. Clustering of NW is favorable because it leads to decreased mutual shielding effect by better separation of emitters [11], and improved current carrying capability by sharing of FE current between many individual NW and improved heat conduction. It should be mentioned that some of the NW are laying flat on the substrate (especially for cathode E with 
long dense NW). This is caused most probably by the wet template dissolution due to insufficient contact strength of NW to the substrate.

Low resolution FESM voltage maps of the whole structured Au-NW cathodes confirm a fairly homogeneous emission from the patch array regions somewhat limited by a slight unflatness of the surface [14]. Typical medium resolution FESM maps of about $1 \mathrm{~mm}^{2}$ scan area of the cathodes shown in figures 4 and 5 demonstrate that the efficiency and alignment of the FE sites strongly depend on the type of nanostructures in the patches. Solitary NW with low number density provide a moderate FE efficiency with only $32 \%$ of emitting patches at fields up to $50 \mathrm{~V} / \mu \mathrm{m}$ (cathode A, not shown), whereas about $70-90 \%$ of the patches with medium and high number density emit at 30-40 V/ $\mu \mathrm{m}$. Aligned FE for patch arrays with $100 \mu \mathrm{m}$ pitch has been found to be more pronounced for short NW due to unordered cluster creation of long NW resulting in a larger scattering of field enhancement factor. The best result in terms of alignment was reproducibly achieved for arrays with $f=10^{7} \mathrm{~cm}^{-2}$ of short NW (see cathode B in figure 4). Medium resolution FESM maps of patch arrays with $150 \mu \mathrm{m}$ pitch show good alignment for cathodes B and C (figure 5), i.e. for both NW length. In contrast, high NW density $\left(f=10^{8} \mathrm{~cm}^{-2}\right)$ leads to less alignment because of preferred FE from the patch edges due to enhanced mutual shielding. This means that the position of the FE sites might be rather on the circumference instead of the interior of the patches. In order to get structured NW cathodes with sufficient alignment for triode devices, therefore, the pitch should be at least twice the patch diameter.

Further high resolution FESM studies of individual patches (figure 6) confirmed the random distribution of FE sites over a patch. They prove the existence of several competing emitters within a patch, especially for high number density of short NW as expected from the corresponding SEM results. The dominant emitter is not necessarily in the center of a patch and might cause some emission image in the spacing between the patches. Multiple emitters per patch, however, contribute to the high efficiency and might be important for the long-term stability of Au-NW cathodes due to substitution of destructed emitters.

The current-voltage (I-V) curves and the maximum current $I_{\max }$ were locally measured for 10-30 patches per cathode. In general strong processing effects were observed especially during the first voltage rise resulting in a stabilized FE from the patches after few minutes. This might be caused by 
adsorbates effects, nanostructural changes of NW tips and/or movement of loose NW in electric field. Patches with long solitary NW showed some current jumps and finally straight FN behavior (figure 7(a)) up to moderate levels of $I_{\max }<450 \mathrm{nA}$. In contrast, $40 \%$ of the patches with few NW clusters yielded stable I-V curves up to $\mu \mathrm{A}$ current levels (figure $7(\mathrm{~b})$ ). Most of the patches with many NW clusters, however, carried stable currents only up to $50 \mathrm{nA}$, and only a few up to $40 \mu \mathrm{A}$ with big current jumps (figure $7(\mathrm{c})$ ). These results indicate a current limitation or stepwise destruction of solitary or clustered NW [10]. According to electrothermal model calculations performed with Comsol [15], resistive heating of the Au-NW at such current levels can be neglected even for significantly reduced electrical and thermal conductivities as compared to the bulk values [16]. A significant uniform heating $(\Delta \mathrm{T}>200 \mathrm{~K})$ is obtained, however, with assumption of very high contact resistance and/or a strongly reduced contact area, which is suggested from SEM images revealing ring-like contact area [17].

Despite of some remaining instabilities of the I-V curves, finally the local electric onset field $E_{\text {on }}$ for a current of $1 \mathrm{nA}$ and effective field enhancement factor $\beta_{\text {eff }}$ of the patches were derived from the measured data. The lowest $E_{\text {on }}$ of $4 \mathrm{~V} / \mu \mathrm{m}$ was obtained for a patch with well-separated long NW (cathode $\mathrm{A}$ ), but the highest $\beta_{\text {eff }}$ of 630 resulted for a patch with many NW clusters (cathode E). The hyperbolic correlation between the $E_{\text {on }}$ and $\beta_{\text {eff }}$ values expected for constant emission area was more pronounced for solitary than for clustered NW [14]. The mean value of $\beta_{\text {eff }}$ and $I_{\max }$ determined for all type of patches scatters significantly and seems to be independent of the aspect ratio as shown in figure 8. The smallest spread of both figures of merit is achieved for short Au-NW of medium number density, while the largest spread results for many competing NW clusters. The correlation between the local $\beta_{\text {eff }}$ and $I_{\max }$ values found for random unstructured Au-NW ensembles with $A R=110$ [11] could not be confirmed for the NW patches. These results reflect the complex interplay of the shape, number density and cluster formation of NW on the FE homogeneity and current carrying capability of Au-NW patches. The mutual shielding effects within the NW patches have a strong influence on their field enhancement as well known for densely grown CNT [18].

Finally we have started to look for correlations between the measured I-V curves and the resulting SEM images of selected Au-NW patches in order to understand an origin of current limitation of the NW. In figure 9 examples of processed patches showing the typically observed destruction are 
compared for the two types of nanostructures. The tips of individual NW were obviously destroyed by successive melting which results in spherically shaped tips and probably explains the rather moderate emission currents. In contrast, the NW clusters provide a better mechanical stability and a higher maximum current because of the mutual support and only partial melting of the NW tips. It is most remarkable that many of the cluster-forming NW in figure 9(b) reveal some ripples on their surface which might reflect thermally driven morphological changes much below the bulk melting temperature (Rayleigh instability) [19], thus proving their contribution to the total current of the cluster. With respect to the electrothermal model calculations mentioned above, these melting features indicate a rather large contact resistance between the NW and the substrate. Unfortunately the contact resistance can not be directly measured because it is difficult to ensure good electrical contact between a wire and a tip anode as a probe without mechanical destruction of the wire. Moreover, in-situ high resolution SEM control would be required which can not be performed within the FESM. Instead, correlated SEM/FESM/SEM studies of single patches are planned to reveal nanostructural changes as function of the FE current.

\section{Conclusions}

The systematic FE measurements for the various types of structured Au-NW cathodes have given valuable hints to optimize regular patch arrays of random nanostructures for triode applications. The best result in terms of efficiency and alignment of the FE sites was achieved for double-spaced patches of $50 \mu \mathrm{m}$ diameter with $\mathrm{NW}$ at number densities of $10^{7} \mathrm{~cm}^{-2}$. Multiple nanostructures per patch contribute to the high emission efficiency of the cathodes. High field enhancement and low onset fields are fairly well correlated for solitary NW, but the achievable maximum currents vary strongly for both types of nanostructures. First correlation studies between FESM maps and SEM images of selected patches revealed the partial melting of solitary and cluster NW as main cause for the obtained current limits. Thermal model calculations and FESM-SEM correlation studies revealed contact problems as main source of NW heating. These results have given suitable hints for a modification of the fabrication steps to improve the FE homogeneity, current strength and stability of structured AuNW cathodes. 


\section{Acknowledgements}

The financial support of GSI for A.N. and V.S. is gratefully acknowledged. We also thank R. Heiderhoff at the Electrical Engineering Department of BUW for support with HRSEM. 


\section{References}

1. W. I. Milne, K. B. K. Teo, G. A. J. Amaratunga, P. Legagneux, L. Gangloff, J. P. Schnell, V. Semet, V. Thien Binh and O. Groening, J. Mater. Chem. 14, p. 933-943 (2004) and ref. therein.

2. N. S. Xu and S. E. Huq, Mater. Sci. Eng. R48, p. 47-189 (2005) and ref. therein.

3. D. S. Y. Hsu and J. L. Shaw, J. Appl. Phys. 98, 014314 (2005).

4. H. M. Manohara, M. J. Bronikowski, M. Hoenk, B. D. Hunt, and P. H. Siegel, J. Vac. Sci. Technol. B 23, 157 (2005).

5. J. H. Lee, S. H. Lee, W. S. Kim, H. J. Lee, J. N. Heo, T. W. Jeong, C. H. Choi, J. M. Kim, J. H. Park, J. S. Ha, H. J. Lee, J. W. Moon, M. A. Yoo, J. W. Nam, S. H. Cho, T. I. Yoon, B. S. Kim, and D. H. Choe, J. Vac. Sci. Technol. B 23, 718 (2005).

6. M. E. Toimil-Molares, J. Brötz, V. Buschmann, D. Dobrev, R. Neumann, R. Scholz, I. U. Schuchert, C. Trautmann, and J. Vetter, Nucl. Instr. Meth. Phys. Res. B 185, 192 (2001).

7. L. Vila, P. Vincent, L. Dauginet-De Pra, G. Pirio, E. Minoux, L. Gangloff, S. DemoustierChampagne, N. Sarazin, E. Ferain, R. Legras, L. Piraux, and P. Legagneux, Nano Lett. 4, 521 (2004).

8. J. Liu, J. L. Duan, M. E. Toimil-Molares, S. Karim, T. W. Cornelius, D. Dobrev, H. J. Yao, Y. M. Sun, M. D. Hou, D. Mo, Z. G. Wang, and R. Neumann, Nanotechnology 17, 1922 (2006).

9. F. Maurer, A. Dangwal, D. Lysenkov, G. Müller, M. E. Toimil-Molares, C. Trautmann, J. Brötz, and H. Fuess, Nucl. Instr. Meth. Phys. Res. B 245, 337 (2006).

10. A. Dangwal, G. Müller, F. Maurer, J. Brötz, and H. Fuess, J. Vac. Sci. Technol. B 25, 586 (2007).

11. A. Dangwal, C. S. Pandey, G. Müller, S. Karim, T. W. Cornelius, and C. Trautmann, Appl. Phys. Lett. 92, 063115 (2008).

12. D. Lysenkov and G. Mueller, Int. J. Nanotechnology 2 (2005) 239.

13. D. Y. Zhong, G. Y. Zhang, S. Liu, T. Sakurai, and E. G. Wang, Appl. Phys. Lett. 80, 506 (2002).

14. A.Navitski, G. Mueller, T.W. Cornelius, C. Trautmann, Technical Digest of $21^{\text {st }}$ Int. Vacuum Nanoelectronics Conf., Wroclaw, p. 75 and EM5 on CD (2008).

15. COMSOL Multiphysics ${ }^{\circledR} 3.5 \mathrm{a}$, www.comsol.com.

16. S. Karim, Dissertation, Chem. Dep. Univ. Marburg, Germany (2007). 
17. A. Navitski, V. Sakharuk, F. Jordan, G. Müller, S. Müller, M. Rauber, M.E. Toimil-Molares, C. Trautmann, Technical Digest of $22^{\text {st }}$ Int. Vacuum Nanoelectronics Conf., Hamamatsu, p. 137 (2009).

18. L. Nilsson, O. Groening, C. Emmenegger, O. Kuettel, E. Schaller, L. Schlapbach, H. Kind, J. M. Bonard, and K. Kern, Appl. Phys. Lett. 76, 2071 (2000).

19. S. Karim S, M. E. Toimil-Molares, A. G. Balogh, W. Ensinger, T. W. Cornelius, E. U. Khan, and R. Neumann, Nanotechnology 17, 5954 (2006). 


\section{Table caption}

Table 1. Overview of parameters of the structured Au-NW cathodes: NW site density $f$, array pitch $P$, nanowire diameter $\mathrm{D}$, length $L$ and aspect ratio $A R$.

\section{Figure captions}

Figure 1. Schematic of the structured cathode fabrication: a) irradiation of polycarbonate foil (white, cut is shown for clarity) with heavy ions (red arrows) through the metallic mask (grey); b) chemical etching of ion tracks; c) deposition of metallic backing layers (orange); d) electrochemical filling of the pores with $\mathrm{Au}$ nanowires (yellow); e) dissolution of polycarbonate template.

Figure 2. SEM images of the metallic mask with $50 \mu \mathrm{m}$ diameter holes in a square array with $100 \mu \mathrm{m}$ pitch and resulting Au-NW patch array.

Figure 3. Typical SEM images of single patches of Au-NW of all cathodes (A-E correspond to a-e) cathodes. The white scale bars corresponds to $10 \mu \mathrm{m}$.

Figure 4. Regulated voltage maps $V(x, y)$ for $1 \mathrm{nA}$ current of cathodes B, C $\left(10^{7} \mathrm{NW} / \mathrm{cm}^{2} ; \mathrm{a}, \mathrm{b}\right)$ and D, $\mathrm{E}\left(10^{8} \mathrm{NW} / \mathrm{cm}^{2} ; \mathrm{c}, \mathrm{d}\right)$ cathodes with short $(\mathrm{a}, \mathrm{c})$ and long $(\mathrm{b}, \mathrm{d}) \mathrm{NW}$ in narrow spaced arrays $(P$ $=100 \mu \mathrm{m}$, scanning anode tip $\left.\emptyset_{\mathrm{a}}=30 \mu \mathrm{m}, \mathrm{d} \approx 20 \mu \mathrm{m}\right)$.

Figure 5. $V(x, y)$ for $1 \mathrm{nA}$ of cathodes $\mathrm{B}, \mathrm{C}\left(10^{7} \mathrm{NW} / \mathrm{cm}^{2}\right)$ with short (a) and long (b) NW in widespaced patch arrays $\left(P=150 \mu \mathrm{m}, \varnothing_{\mathrm{a}}=30 \mu \mathrm{m}, \mathrm{d} \approx 20 \mu \mathrm{m}\right)$.

Figure 6. High resolution $V(x, y)$ for $1 \mathrm{nA}$ of patch arrays of short Au-NW: (a) cathode $\mathrm{B}, f=10^{7} \mathrm{~cm}^{-2}$, $P=150 \mu \mathrm{m}, \varnothing_{\mathrm{a}}=5 \mu \mathrm{m}$; (b) cathode D, $f=10^{8} \mathrm{~cm}^{-2}, P=100 \mu \mathrm{m}, \varnothing_{\mathrm{a}}=3 \mu \mathrm{m}$.

Figure 7. Typical I-V curves and FN plots (insets) of single patches with long Au-NW for $f=10^{6} \mathrm{~cm}^{-2}$ of cathode $\mathrm{A}(\mathrm{a}), 10^{7} \mathrm{~cm}^{-2}$ of cathode $\mathrm{C}(\mathrm{b})$ and $10^{8} \mathrm{~cm}^{-2}$ of cathode $\mathrm{E}$ (c) measured with $\emptyset_{\mathrm{a}}=$ $30 \mu \mathrm{m}$ at $d \approx 20 \mu \mathrm{m}$. Please note the different current scales. Arrows indicate increase of voltage (1), current drop due to full or partial destruction of a FE site (2) and decrease of voltage (3).

Figure 8. Mean value (dots) and range (bars) of measured field enhancement factor $\beta_{\text {eff }}$ and maximum stable emission current $\mathrm{I}_{\max }$ for all patch types vs. aspect ratio of Au-NW. 
Figure 9. Typical SEM images of selected Au-NW patches $\left(f=10^{8} \mathrm{~cm}^{-2}, L=7 \mu \mathrm{m}\right)$ : destruction of single NW at $I \approx 3 \mu \mathrm{A}$ (a) and NW clusters at $I \approx 90 \mu \mathrm{A}$ (b). 


\begin{tabular}{c|c|c|c|c|c|} 
Cathode & A & B & C & D & E \\
\hline$f\left[\mathrm{~cm}^{-2}\right]$ & $10^{6}$ & \multicolumn{2}{|c|}{$10^{7}$} & \multicolumn{2}{|c|}{$10^{8}$} \\
\hline$P[\mu \mathrm{m}]$ & 150 & \multicolumn{2}{|c|}{100} & \multicolumn{2}{|c|}{100} \\
\hline$D[\mathrm{~nm}]$ & 311 & \multicolumn{2}{|c|}{270} & \multicolumn{2}{|c|}{270} \\
\hline$L[\mu \mathrm{m}]$ & 25 & 8 & 28 & 7 & 28 \\
& \pm 3 & \pm 1 & \pm 2 & \pm 1 & \pm 2 \\
\hline $\mathrm{AR}$ & 161 & 60 & 207 & 52 & 207
\end{tabular}

Table 1. 

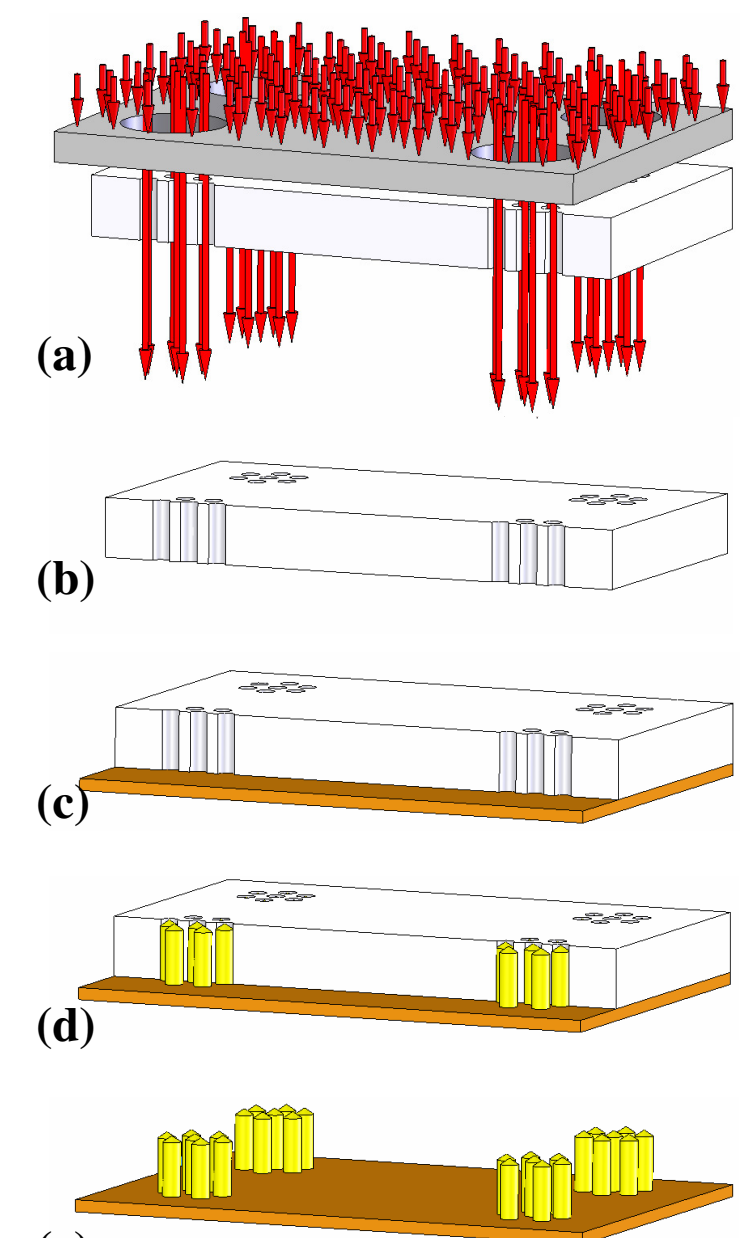

(e)

Figure 1. 


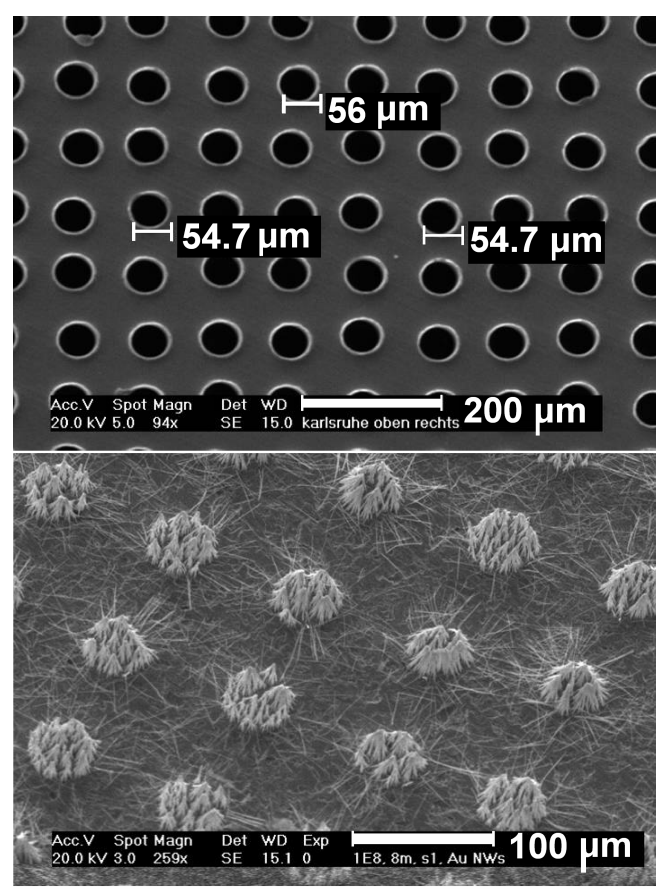

Figure 2. 

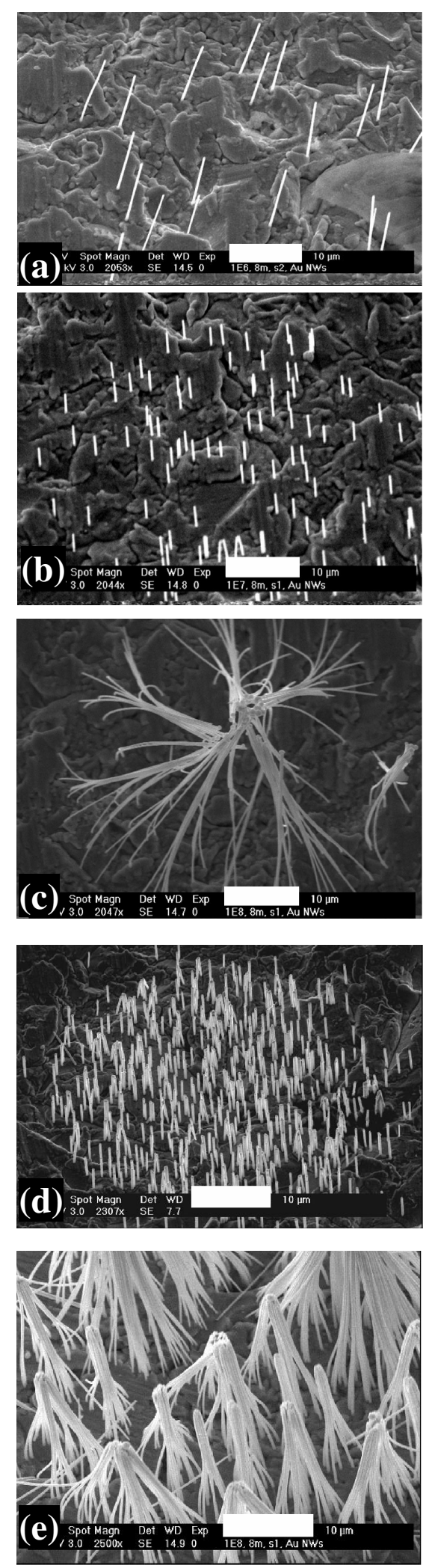

Figure 3. 


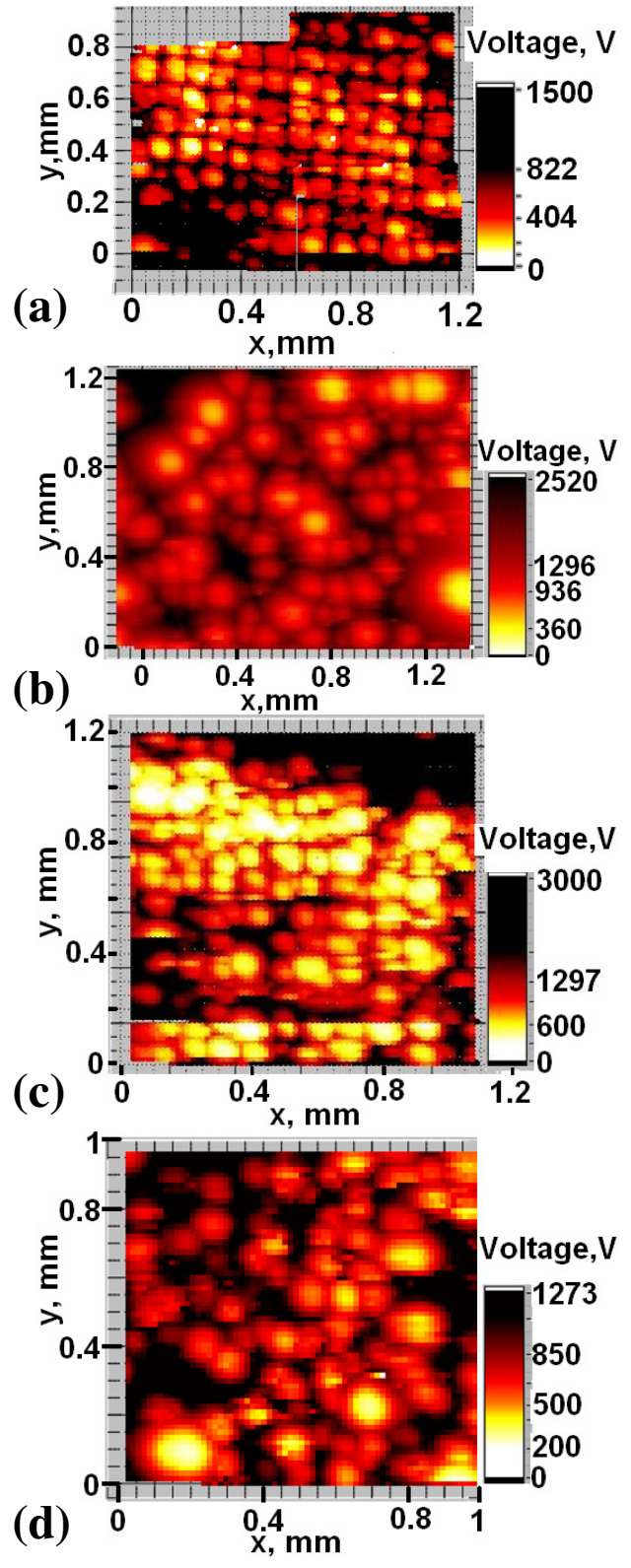

Figure 4. 


\section{Figure 5.}

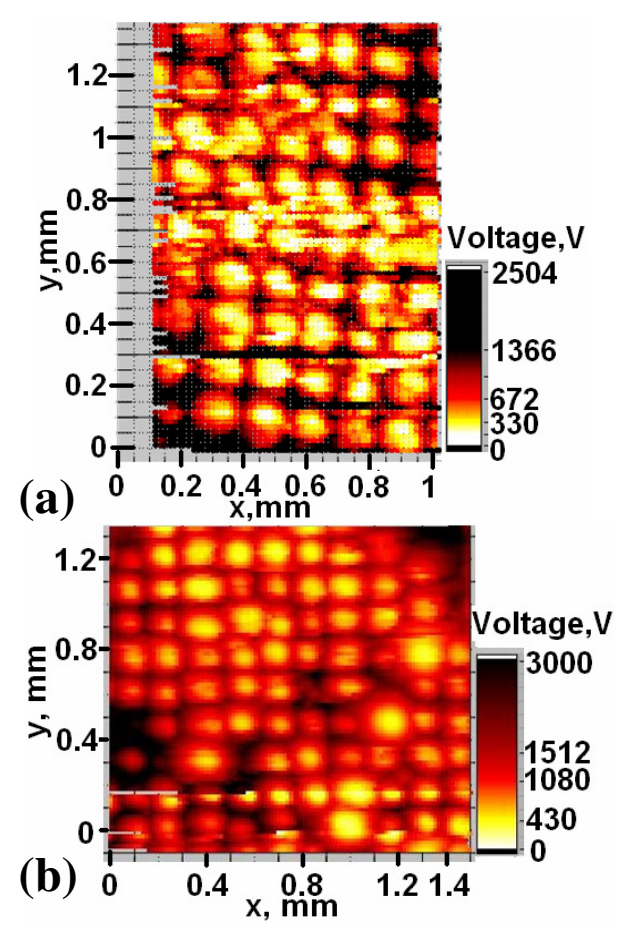



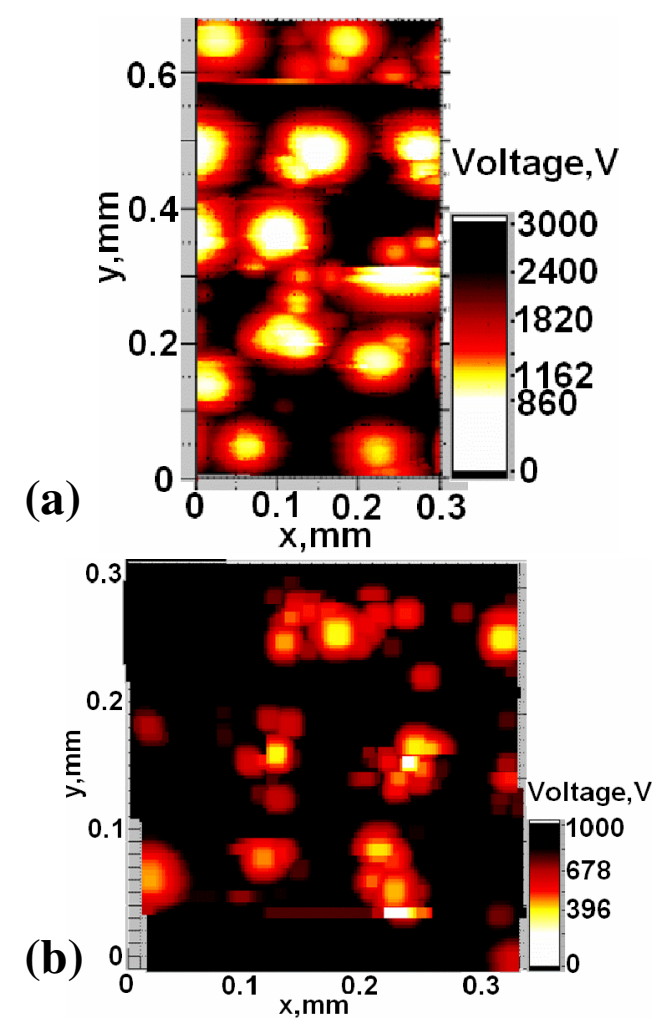

Figure 6. 

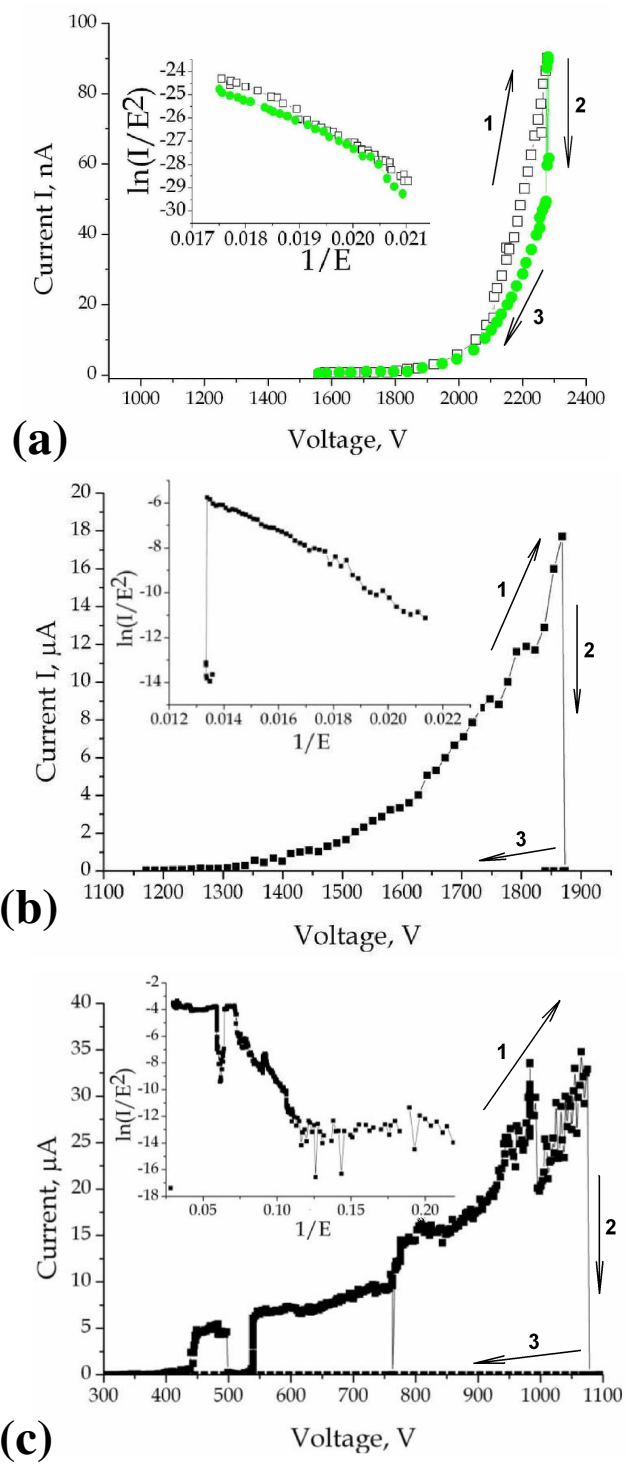

Figure 7. 


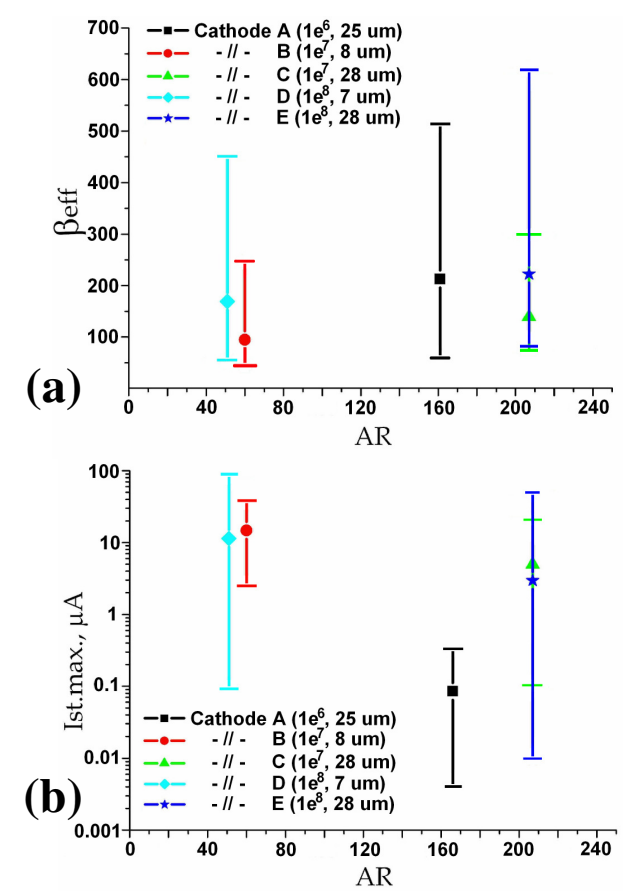

Figure 8. 


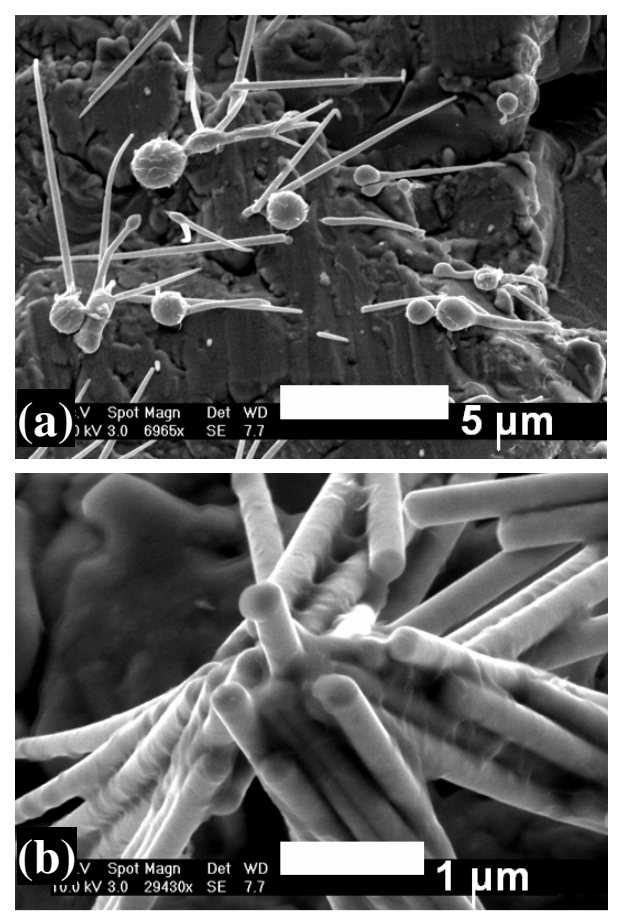

Figure 9. 